\title{
Novel mutations identified in Chinese families with autosomal dominant congenital cataracts by targeted next- generation sequencing
}

\author{
Shan $\mathrm{Li}^{1+}$, Jianfei Zhang ${ }^{2 \dagger}$, Yixuan Cao ${ }^{1}$, Yi You $^{1}$ and Xiuli Zhao ${ }^{1 *}$ (D)
}

\begin{abstract}
Background: Congenital cataract is a clinically and genetically heterogeneous visual impairment. The aim of this study was to identify causative mutations in five unrelated Chinese families diagnosed with congenital cataracts.

Methods: Detailed family history and clinical data were collected, and ophthalmological examinations were performed using slit-lamp photography. Genomic DNA was extracted from peripheral blood of all available members. Thirty-eight genes associated with cataract were captured and sequenced in 5 typical nonsyndromic congenital cataract probands by targeted next-generation sequencing (NGS), and the results were confirmed by Sanger sequencing. Bioinformatics analysis was performed to predict the functional effect of mutant genes.

Results: Results from the DNA sequencing revealed five potential causative mutations: c.154T > C(p.F52 L) in GJA8 of Family 1, c.1152_1153insG(p.S385Efs*83) in GJA3 of Family 2, c.1804 G > C(p.G602R) in BFSP1 of Family 3, c.1532C > T(p.T511 M) in EPHA2 of Family 4 and c.356G > A(p.R119H) in HSF4 of Family 5. These mutations co-segregated with all affected individuals in the families and were not found in unaffected family members nor in 50 controls. Bioinformatics analysis from several prediction tools supported the possible pathogenicity of these mutations.

Conclusions: In this study, we identified five novel mutations (c.154 T > C in GJA8, c.1152_1153insG in GJA3, c.1804G > C in BFSP1, c.1532C > T in EPHA2, c.356G > A in HSF4) in five Chinese families with hereditary cataracts, respectively. NGS can be used as an effective tool for molecular diagnosis of genetically heterogeneous disorders such as congenital cataract, and the results can provide more effective clinical diagnosis and genetic counseling for the five families.
\end{abstract}

Keywords: Congenital cataract, Next-generation sequencing, Gene mutation, Bioinformatics analysis

\section{Background}

Congenital cataract is a clinically and genetically heterogeneous lens disorder, characterized by opacification of crystalin lens at birth or during early childhood [1]. The prevalence of congenital cataracts varies from 1 to 6 per 10,000 live births [2]. Approximately one third of the cases have a family history [3]. The cataract may be an isolated anomaly, or part of a multisystem syndrome [4].

\footnotetext{
* Correspondence: xiulizhao@ibms.pumc.edu.cn

†'Shan Li and Jianfei Zhang contributed equally to this work.

'Department of Medical Genetics, Institute of Basic Medical Sciences Chinese

Academy of Medical Sciences - School of Basic Medicine Peking Union

Medical College, 5 Dong Dan San Tiao, Dongcheng District, Beijing 100005,

People's Republic of China

Full list of author information is available at the end of the article
}

Both X-linked and autosomal recessive inheritance patterns have been reported for congenital cataract, however autosomal dominant trait is the most prevalent mode [5-7]. Cataracts can be classified as sutural, whole lens, nuclear, lamellar, cortical, polar, cerulean, coralliform, and other subtypes, according to morphology of lens $[8-10]$.

To date, at least 30 pathogenic genes have been found to link to congenital cataracts. From the reported mutant genes in congenital cataract families, nearly half of the mutations associated with crystalin genes [11], including genes coding for crystalin families (CRYAA, OMIM 604219; CRYAB, OMIM 613763; CRYBA1, OMIM 600881; CRYBB1, OMIM 611544; CRYBB2, OMIM 601547; CRYBB3, OMIM 609741;

(c) The Author(s). 2019 Open Access This article is distributed under the terms of the Creative Commons Attribution 4.0 International License (http://creativecommons.org/licenses/by/4.0/), which permits unrestricted use, distribution, and 
CRYGC, OMIM 604307; CRYGD, OMIM 115700; CRYGS, OMIM 116100), gap junctional proteins (GJA3, OMIM 601885; GJA8, OMIM 116200), beaded filament structural proteins (BFSP1, OMIM 611391; BFSP2, OMIM 611597), and other functional genes (e.g., HSF4, OMIM 116800; MIP, OMIM 615274; PITX3, OMIM 610623; EPHA2, OMIM 116600) [7, 9, 12-15].

Identification of accurate genetic cause of congenital cataract is essential for providing precise diagnosis and genetic counseling [8]. However, due to the high clinical and genetic heterogeneities, clinical and genetic diagnostic of congenital cataract, especially for nonsyndromic congenital cataracts, are limited by the traditional sequencing method, by which only few candidate genes can be sequenced at each time [16]. Recently, the next generation sequencing (NGS) combined with targeted genomic enrichment has proved to be an effective solution to the genetic test of genetically heterogeneous diseases and provides a new opportunity for genetic diagnostics of congenital cataracts [12, 17].

In this study, we collected information from five large Chinese families with congenital cataracts. Then we performed targeted enrichment and deep sequencing to detect the genetic mutations in these families. We identified five novel mutations in the GJA3 (S385Efs"83), GJA8 (F52 L), BFSP1 (G602R), EPHA2 (T511 M) and HSF4 (R119H) genes that potentially resulted in the development of congenital cataract. With Sanger sequencing, we confirmed that mutations were co-segregated with affected individuals in the five families, whereas mutations were not found in unaffected family members and normal controls. Bioinformatics analysis, conservative prediction and 3-D protein simulation indicated that the five mutations might be the pathogenic mutations for congenital cataract families. This study demonstrates that the targeted gene sequencing can be used as an effective tool for genetics diagnosis of congenital cataract.

\section{Materials and methods}

Clinical examination and isolation of genomic DNA

Five Chinese pedigrees with autosomal dominant hereditary cataract were collected from The No.4 hospital (eye hospital) of Zhangjiakou, Hebei, China, and 50 unrelated subjects without eye diseases were enrolled as normal controls. Informed written consents were obtained from all adult participants and the legal guardians of children under age 18 and $3-5 \mathrm{~mL}$ peripheral blood samples were collected from all available members. Affected individuals were confirmed by histories of cataract surgery or ophthalmological examinations, and their clinical phenotypes were recorded by slit-lamp photography. Genomic DNA was extracted from peripheral blood using standard SDS-proteinase K-phenol/chloroform method [18]. This study was approved by the Institutional Review
Board (IRB) of the Institute of Basic Medical Sciences, Chinese Academy of Medical Sciences, Beijing, China (015-2015).

\section{Targeted capturing and next generation sequencing}

A capture array (NimbleGen, Roche) was designed to capture all exons, splice sites and adjacent introns sequences of 38 known pathogenic genes associated with inherited cataract diseases based on GeneReviews (NCBI) [12, 19] (Additional file 1: Table S1). Genomic DNA was fragmented ranging from $200 \mathrm{bp}$ to $250 \mathrm{bp}$ and purified, followed by treatment with T4 DNA polymerase, T4 phosphonucleotide kinase and Klenow fragment of DNA polymerase to fill $5^{\prime}$ overhangs and to remove $3^{\prime}$ overhangs. According to standard Illumina protocols, terminal A residues were added following a brief incubation with the Klenow 3' -5 ' exo-enzyme and dATP. Adapter oligonucleotides from Illumina (single reads) were ligated to the ends. Subsequently, ligation was confirmed by four-cycle PCR using a high-fidelity polymerase with primers containing a customsynthesized barcode sequence ( $8 \mathrm{bp}$ ) as a sample index signature. PCR generated a library for further analysis, and the indexed fragments and DNA adapter-ligated were pooled and hybridized to the capture array. After hybridization and enrichment, the DNA sample was sequenced on Illumina HiSeq2000 Analyzers to generate paired end reads (90 bps) [17]. Raw data was generated by Illumina Pipeline, followed by imaging analysis and base calling. Short-reads mapping was then mapped to the human genome reference from the NCBI database (Build 37) using the Multi-Vision software package of Burrows Wheeler Aligner. Single nucleotide variants (SNVs) were determined by SOAPsnp, and small insertion and deletions (InDels) were identified using the GATK InDel Genotyper. Previously identified SNPs were determined using the NCBI dbSNP (http://www.ncbi. nlm.nih.gov/SNP/) or HapMap databases (http://hapmap.ncbi.nlm.nih.gov/). Known disease-causing mutations were identified from the Human Gene Mutation Database HGMD (http://www.hgmd.org/) or from mutations reported previously. All reference sequences were based on the NCBI37/hg19 assembly of the human genome.

\section{Sanger sequencing}

To validate the DNA variants (substitutions or indels) generated from next-generation sequencing, the target sites and their flanking sequences were examined by PCR combined with Sanger DNA sequencing in the corresponding proband. Genomic DNA reference sequences of GJA8 (NM 005267.4), GJA3 (NM_021954.3), BFSP1 (NM_001195.3), EPHA2 (NM_004431.3) and HSF4 (NM_001538.3) were obtained from the University of California, Santa Cruz (UCSC) 
Genome Browser database (http://genome.ucsc.edu/). All primers for PCR were designed via online tool Primer3 (http://primer3.ut.ee/) (Table 1). Sanger sequencing was then performed in all probands and unaffected family members. The PCR program was performed as following: $95^{\circ} \mathrm{C}$ for 3 min; $94{ }^{\circ} \mathrm{C}$ for $30 \mathrm{~s}, 58^{\circ} \mathrm{C}$ for $30 \mathrm{~s}, 72^{\circ} \mathrm{C}$ for $40 \mathrm{~s}(38$ cycles); $72{ }^{\circ} \mathrm{C}$ for $8 \mathrm{~min}$. The PCR products were separated by $2 \%$ agarose gel electrophoresis, and the target fragment was purified by the QIAquick Gel Extraction kit (Qiagen, Hilden, Germany) according to the manufacturer's protocol. Sequencing result from Applied Biosystems 3730xl DNA Analyzer (Thermo Fisher Scientific, Waltham, MA, USA) was aligned to reference sequence through CodonCode Aligner (version 6.0.2.6; CodonCode, Centerville, MA, USA).

\section{Bioinformatics analysis}

The amino acid sequences of protein Gap junction alpha- 8 protein (encoded by GJA8), Filensin (encoded by BFSP1), Ephrin type-A receptor 2 (encoded by EPHA2), and Heat shock factor protein 4 (encoded by HSF4) were obtained from NCBI Protein database (FASTA format). Multiple sequence alignments with different animals (humans, mouse, chicken, monkey, snake, frog and zebrafish) and conservative analysis were performed by the software MEGA (Version7; Institute for Genomics and Evolutionary Medicine, Temple University, USA). Functional effects of the mutations was predicted by Online tools Polymorphism Phenotyping version2 (PolyPhen-2; http://genetics.bwh.harvard.edu/pph2/), Scale-Invariant Feature Transform (SIFT; http://sift.jcvi.org/), Protein Variation Effect Analyzer (PROVEAN; http://provean. jcvi.org/seq_submit.php), Mutation Taster (http://www. mutationtaster.org) and M-CAP (http://bejerano.stanford.edu/mcap/) to assess the possible effects of variants on protein structure and function, regarding sequence conservation, chemical change, and likelihood of pathogenicity. 3D structures of normal and missense mutants in Gap junction alpha-8 protein (PDB: 6MHY), Ephrin type-A receptor 2 (PDB: 2X10) and Heat shock factor protein 4 (PDB: 2IDU) were generated by homology modeling using SWISS-MODEL (http://swissmodel. expasy.org/), however, because wild-type human filensin hadn't been crystallized and there was no homologous sequence of filensin, a 3D model of filensin couldn't be generated. The interactions between the amino acid and the neighboring residues were exhibited and simulated by PyMOL (Schrödinger, LLC, New York, NY, USA; http://www.pymol.org/).

\section{Results \\ Clinical evaluation \\ Family 1}

Family 1 had two-generations, including two affected and three unaffected individuals (Fig. 1a). All patients in this family presented bilateral nuclear cataracts with white pupil at birth (Table 2). Nucleus density of lens increased with age presenting a gray opacity, however without other noticeable unusual eye structure. Slitlamp examination revealed dense nuclear cataract in the center in the II1 (Fig. 2a). All patients were treated using phacoemulsification combined with intraocular lens implantation surgery. The postoperative visual acuity was good and the corrected visual acuity reached 0.5 .

\section{Family 2}

Five members in a two-generation Chinese family with a history of cataracts participated in the study, including three affected and two unaffected individuals (Fig. 1b). Slit-lamp photograph of proband (II1) showed a nuclear/ lamellar cataract with blue punctate opacities (Fig. 2b). All patients with lamellar cataract presented high density of crystal nucleus and crystal density, both of which increased along with age. There was no evidence of other ocular or systemic defects. These features were similar among all the affected participants (Table 2).

\section{Family 3}

Nine family members of a three-generation Chinese family with a history of cataracts participated in the study, including five affected and four unaffected individuals (Fig. 1c). All patients emerged visible white pupil at birth and crystal gray opacity (Table 2). All patients in this family had bilateral cataracts. The pupils of patient (III3) were white at birth, presented the overall gray crystal (Fig. 2c). While double front sections, intraocular pressures and vitreous body did not show any abnormality.

Table 1 Primers for PCR and Sanger sequencing used in mutation validation

\begin{tabular}{llllll}
\hline Gene & Transcript & mutation & Forward primer (5'-3') & Reverse primer (5'-3') & Product length (bp) \\
\hline GJA8 & NM_005267.4 & c.154T>C & GCAACTTGGAAAGGAGAGGT & ATGTGGCAGATGTAGGTCCT & 576 \\
GJA3 & NM_021954.3 & c.1152_1153insG & GATGACTGAGCAGAACTGGG & CCTGATCTCTCCTCCATCGT & 496 \\
BFSP1 & NM_001195.3 & c.1804G >C & CCAATTGACCAGCAGCCTAT & CTGTCCTCATGAAGCTGACC & 623 \\
EPHA2 & NM_004431.3 & c.1532C > T & AATTCCGAGCCTCAGTTCC & TGAACTTCCTCACACCACTG & 540 \\
HSF4 & NM_001538.3 & c. 356G > A & CTGCCCCAGTATTTCAAGCA & CCTCCTCTTGCTCATTCCC & 309 \\
\hline
\end{tabular}




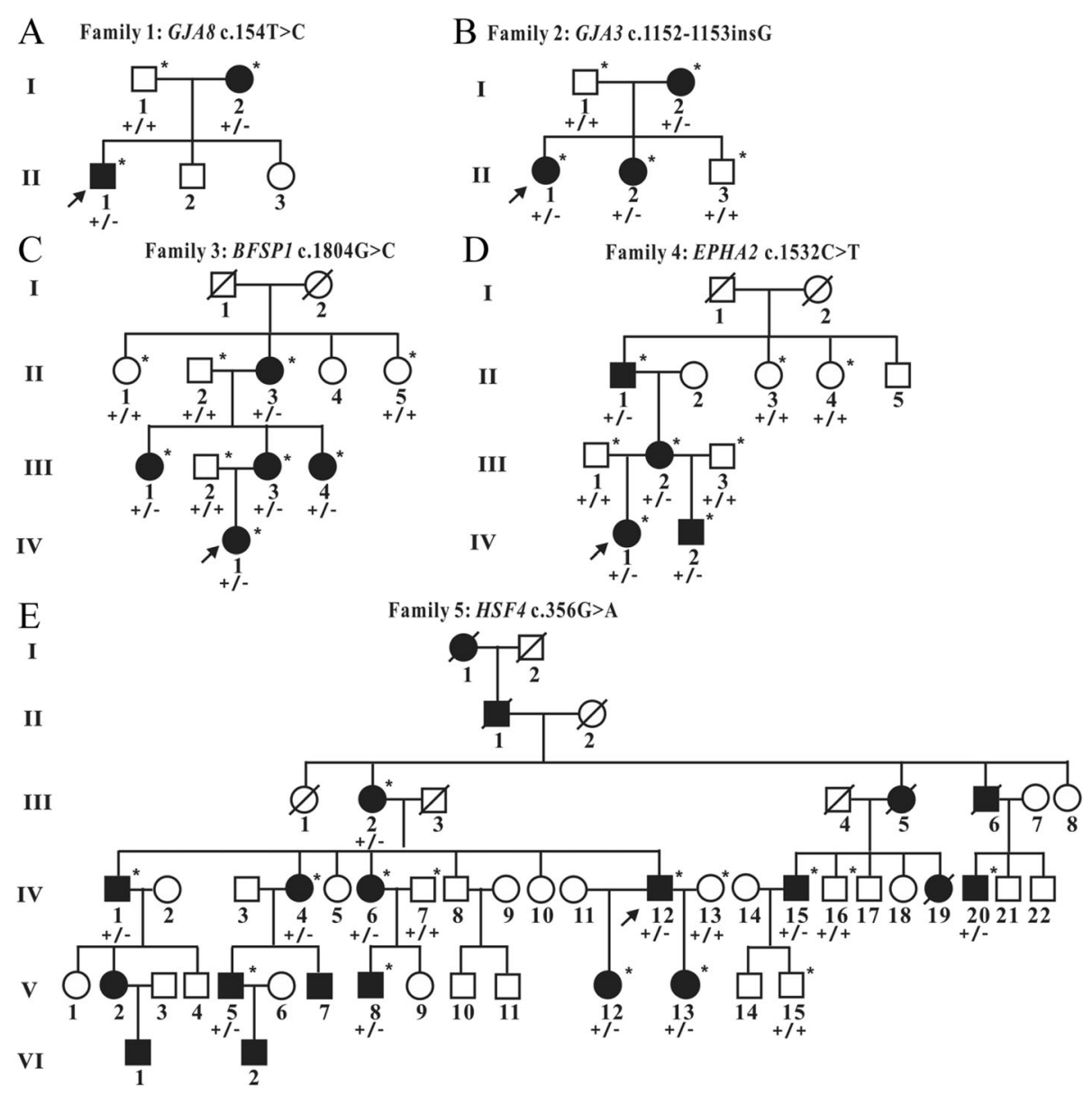

Fig. 1 The pedigrees and genotypes of 5 Chinese families with congenital autosomal dominant cataracts. The probands are indicated with an arrow. Squares and circles symbolize male and female individuals respectively. Black symbols indicate affected members and blank symbols indicate unaffected individuals. Asterisks indicate sequenced samples. " $+/+$ " indicates wild-type and " $+/-^{\prime \prime}$ indicates heterozygote. (a) Pedigree of Family 1, all patients carried the heterozygous mutation c.154 T > C in GJA8. (b) Pedigree of Family 2, all patients carried the heterozygous mutation C.1152_1153insG in GJA3. (c) Pedigree of Family 3, all patients carried the heterozygous mutation c.1804G > C in BFSP1. (d) Pedigree of Family 4, all patients carried the heterozygous mutation C.1532C > T in EPHA2. (E) Pedigree of Family 5, all patients carried the heterozygous mutation c.356G > A in HSF4

\section{Family 4}

Eight family members of a three-generation Chinese family with a history of cataracts participated in the study, including four affected and four unaffected individuals (Fig. 1d). The opacities of proband (IV1) were visible at birth, which had a great influence on visual acuity. She was diagnosed with bilateral total cataract and presented nuclear opacity. These features were similar among all the affected participants (Table 2). There was no evidence of other systemic or ocular defects with the affected family members.

\section{Family 5}

This family included 9 affected females, 11 affected males and 31 unaffected members in a six-generation pedigree (Fig. 1e). All the affected members in this family were diagnosed as zonular and nuclear cataract coupled with increased crystal nucleus density and they had poor eyesight during the child period of 3 6 months (Table 2). There were no other ocular abnormalities nor other systematic diseases with the patients. The proband (IV12) showed a nuclear cataract (Fig. 2d) and received an operation at age 21 with YAG laser release incision of posterior capsular. Postoperative vision of the proband reached up to 0.6 , and vision condition gradually improved after amblyopia training.

\section{Identification of mutations}

Thirty-eight genes (Additional file 1: Table S1) related with inheritable and congenital cataract were captured and sequenced by next-generation sequencing. The average coverage was approximately $99.2 \%$, and the average median depth was $475 \times .100 \%$ of base pairs with N200× coverage was successfully detected indicating high capabilities for 
Table 2 Clinical information of 22 patients from five congenital cataract families

\begin{tabular}{|c|c|c|c|c|c|c|}
\hline Family number & Patient & Gender & Age at onset & Age at diagnosis (year) & Age at surgery (year) & Phenotype of cataract \\
\hline \multirow[t]{2}{*}{ Family 1} & 12 & Female & On birth & 9 & 17 & Nuclear, white opacities \\
\hline & $\| 1$ & Male & On birth & 5 & 6 & Nuclear, white opacities \\
\hline \multirow[t]{3}{*}{ Family 2} & 12 & Female & On birth & 17 & 21 & Nuclear/lamellar cataract, blue punctate opacities \\
\hline & $\| 1$ & Female & On birth & 7 & 9 & Nuclear/lamellar cataract, blue punctate opacities \\
\hline & 112 & Female & On birth & 2 & 7 & Nuclear/lamellar cataract, blue punctate opacities \\
\hline \multirow[t]{5}{*}{ Family 3} & $\| 3$ & Female & On birth & 20 & 20 & Total cataract \\
\hline & $\| 111$ & Female & On birth & 2 & 5 & Total cataract \\
\hline & III3 & Female & On birth & 2 & 3 & Total cataract \\
\hline & $\| 114$ & Female & On birth & 1 & 2 & Total cataract \\
\hline & IV1 & Female & On birth & On birth & 0.25 & Total cataract \\
\hline \multirow[t]{4}{*}{ Family 4} & $\| 1$ & Male & On birth & 10 & 21 & Total cataract \\
\hline & 1112 & Female & On birth & 2 & 17 & Total cataract \\
\hline & IV1 & Female & On birth & On birth & 0.33 & Total cataract \\
\hline & IV2 & Male & On birth & On birth & 0.17 & Total cataract \\
\hline \multirow[t]{8}{*}{ Family 5} & III2 & Female & On birth & 32 & 60 & Nuclear, white opacities \\
\hline & IV1 & Male & On birth & 16 & 20 & Nuclear, white opacities \\
\hline & IV12 & Male & On birth & 12 & 21 & Nuclear, white opacities \\
\hline & V2 & Female & On birth & 2 & 5 & Nuclear, white opacities \\
\hline & V12 & Female & On birth & On birth & 2 & Nuclear, white opacities \\
\hline & V13 & Female & On birth & On birth & 2 & Nuclear, white opacities \\
\hline & VI1 & Male & On birth & On birth & 2 & Nuclear, white opacities \\
\hline & $\mathrm{V} 12$ & Male & On birth & On birth & 2 & Nuclear, white opacities \\
\hline
\end{tabular}
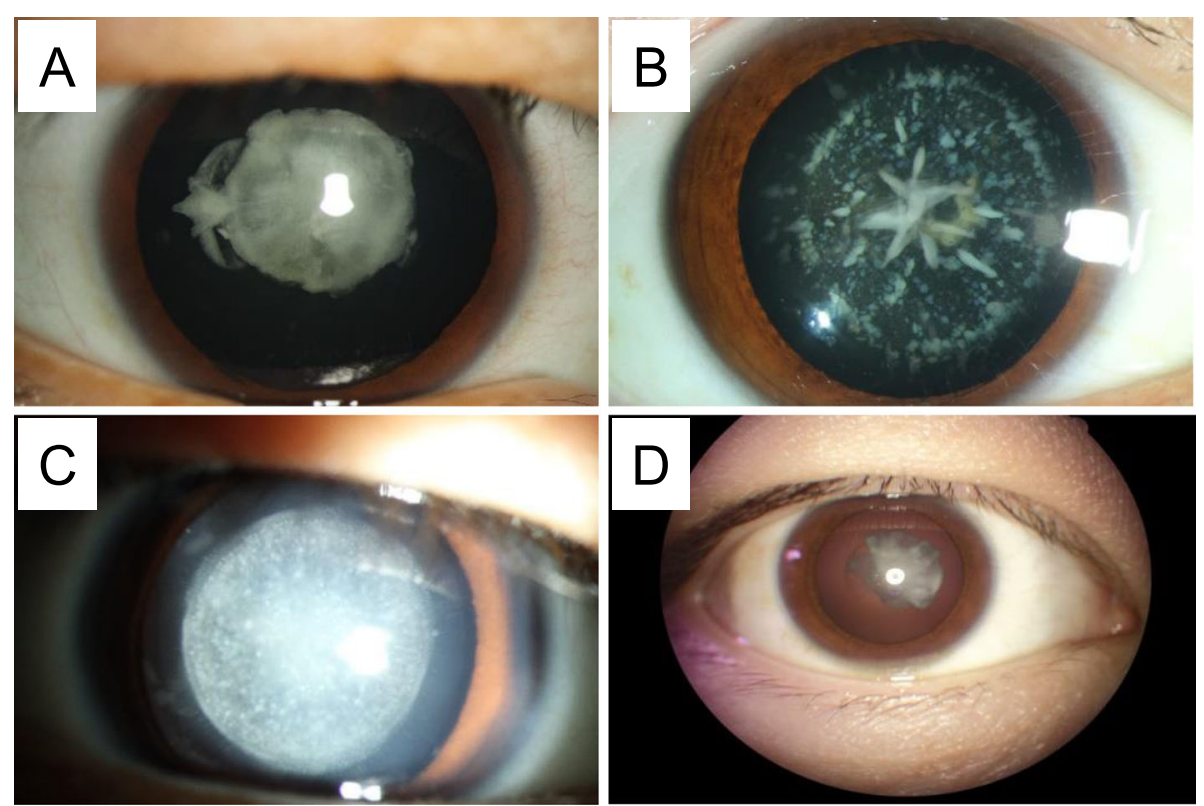

Fig. 2 Slit lamp photographs of the patients from four families. (a) The proband (II1) in the Family 1 presented nuclear cataract. (b) The proband (II1) in Family 2 showed a nuclear/lamellar cataract with blue punctate opacities. (c) The patient (III3) in Family 3 showed a total cataract. (d) The proband (IV12) in Family 5 showed a nuclear cataract 
identifying variants. Variants in five cataract probands from the targeted NGS in Additional file 2: Table S2. The variants were excluded if they presented high frequency in the 1000 Genome database or the dbSNP database. Since the five family pedigrees accorded with autosomal dominant inheritance, we first focused on heterozygous mutations. Five potential pathogenic mutations were confirmed in the five probands associated with congenital cataract: the heterozygous mutation c.154 T > C (p.F52 L) in GJA8 in Family 1, c.1152_1153insG (p.S385Efs"83) in GJA3 in Family 2, and c. 1804G > C (p.G602R) in BFSP1 in Family 3, c.1532C > T (p.T511 M) in EPHA2 in Family 4 and mutation c. 356G > A (p. R119H) in HSF4 in Family 5. The five mutations were novel and were first identified as associated with congenital cataract. The mutations were further confirmed by Sanger sequencing (Fig. 3), and the five mutations co-segregated with the phenotypes in five families (Fig. 1). Additional testing proved that mutations were not detected in 50 healthy local Chinese controls.

\section{Bioinformatics analysis of the mutations}

Conservation analysis of amino acid located in p.F52 of Gap junction alpha-8, p.G602 of Filensin, p.T511 of Ephrin type-A receptor 2, and p.R119 of Heat shock factor protein 4 within different vertebrate species was performed. The analysis indicated that those p.F52 of Gap junction alpha-8, p.T511 of Ephrin type-A receptor 2, and p.R119 of Heat shock factor protein 4 amino acid sites were highly conserved except p.G602 of Filensin, and the replacement of wild type residues might change their biological function (Fig. 4). In addition, SIFT, PolyPhen-2, MutationTaster, M-CAP and PROVEAN programs yielded similar outcomes regarding pathogenicity except that MutationTaster and PROVEAN predicted p.G602R was a neutral mutation (Table 3). According to the SWISS-MODEL prediction, substitution of phenylalanine into a leucine at position 52 of Gap junction alpha- 8 protein would influence the conformation of the protein. In addition, the residue was buried in the core of a domain, and the mutant residue might disturb the core structure of this domain (Fig. 5a). Then, simulation predicted that T511 interacted via $\mathrm{H}$-bonding with residues $\mathrm{N} 435$ and Q515 of Ephrin type-A receptor 2. Substitution of M511 destroyed the $\mathrm{H}$-bonding as the original wild-
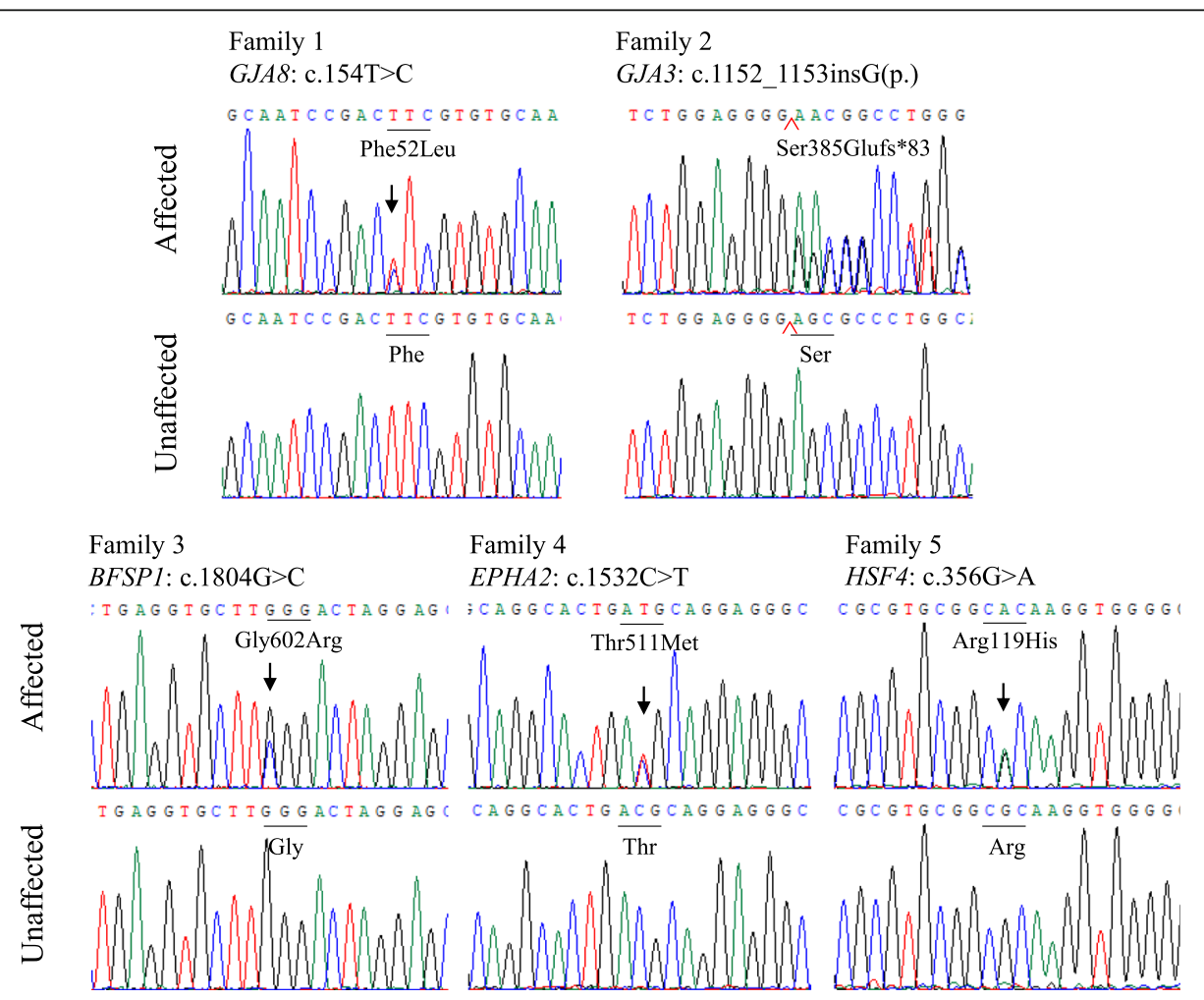

Fig. 3 The potential causative mutations were identified in five Chinese families with congenital cataract. (a) The heterozygous mutation c.154 $\mathrm{T}>\mathrm{C}(\mathrm{p} . \mathrm{F} 52 \mathrm{~L}$ ) in GJA8 was identified in all the affected participants in the Family 1. (b) The heterozygous mutation c.1152_1153insG(p.S385Efs*83) in GJA3 was identified in all the affected participants in the Family 2. (c) The heterozygous mutation c.1804G >C(p.G602R) in BFSP1 was identified in all the affected participants in the Family 3. (d) The heterozygous mutation c.1532C > T(p.T511 M) in EPHA2 was identified in all the affected participants in the Family 4. (e) The heterozygous mutation c.356G > A(p.R119H) in HSF4 was identified in all the affected participants in the Family 5 


\begin{tabular}{|c|c|c|c|c|}
\hline \multirow[b]{2}{*}{ A } & \multicolumn{2}{|r|}{ GJA8: p.(F52L) } & & BFSP1: p.(G602R) \\
\hline & Human & A A EF VWGDEQSDFFV V N Q QPGCEN V & B Human & 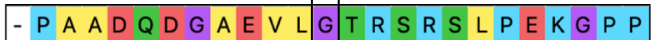 \\
\hline & Mouse & A A E F VWGDEQSD $F / V C N T Q Q P G C E N V$ & Mouse & - $S G S N Q G G P V G P|A| S K S S L L A K G P S$ \\
\hline & Chicken & A A E L VWGDEQSD/F/VCNTQQPGCEN V & Chicken & E T D H D R D KQG L L F R E A G L - . P P \\
\hline & Mo & A A E F VWGDEQSD F/VCNTQQPGCEN V & Monkey & $-P \vee A D Q D G A E G L G T R G R S L P E K G P P$ \\
\hline & Snake & A A E F VWGDEQSD F/VCNTQQPGCEN V & Snake & $\ldots$ KKQCNKQDG|L|HKELAS $\ldots$ P \\
\hline & Frog & A A E F VWGDEQSD F VCNTQQPGVKCL & Frog & - PGANNEYMASQA|VRDADC .... L \\
\hline & & A A E F VWGDEQS D Y V V C N T Q QP G CEN V & Zebrafish & - -GGNKDPSEPPPPMSYSS - I I P P \\
\hline & \multicolumn{2}{|r|}{ EPHA2: p.(T511M) } & & $H S F 4: \mathrm{p} .(\mathrm{R} 119 \mathrm{H})$ \\
\hline \multirow[t]{7}{*}{$\mathrm{C}$} & Human & 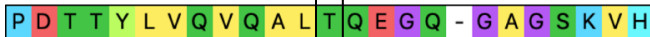 & D Human & 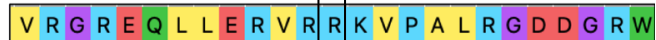 \\
\hline & $\mathrm{M}$ & $P D T T Y L V Q \vee Q A L T Q E G Q-G A G S K V H$ & Mouse & VRGREQLLER VRR K $V P A L R G D D S R W$ \\
\hline & cken & $P D T T Y L V Q V Q A L \mid T Q E G Q-G A G S K V H$ & Chicken & LQGHEHLLEH I K R KV SVVKSEETKM \\
\hline & Monkey & $P G T S Y V \vee R \vee Q A L|T| K D G H-G A F S L E H$ & Monkey & $\vee R G R E Q L L E V R R K \vee P A L R G D D G R W$ \\
\hline & Snake & P G TKY V F R VH T L TAEGHPSSHSAEL & Snake & $\ldots \ldots-\ldots$ - $\ldots$ S V K SE E T KM \\
\hline & Frog & $P G T T Y \vee V R \vee Q A L T Q E G V-G \vee Y S R D Y$ & Frog & LQGHEHLLEH I K R K K V SVVKSEETKM \\
\hline & & $P K T K Y L V S \vee Q A L|T| Q E G H-G A H S L E H$ & & 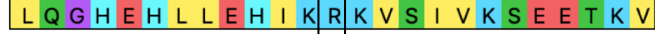 \\
\hline
\end{tabular}

Fig. 4 The multiple-sequence alignments from different vertebrate species. (a-d) The amino acid alterations, F52 L of GJA8 in Family 1, T511 M of EPHA2 in Family 4 and R119H of HSF4 in Family 5 were located in highly conserved region among all vertebrate species and were marked with box. While G602R of BFSP1 in Family 3 had lower conservative propertys

type residue did. (Fig. 5b). Meanwhile, substitution of H119 destroyed the H-bonding, with which wildtype R119 interacted with residues L124 of Heat shock factor protein 4 (Fig. $5 \mathrm{c}$ ). This indicated that the substitution would affect protein function.

\section{Discussion}

We reported five novel mutations associated with the autosomal dominance cataract in five Chinese families respectively: c.154 T > C in GJA8, c.1152_1153insG in GJA3, c. $1804 \mathrm{G}>\mathrm{C}$ in BFSP1, c. $1532 \mathrm{C}>\mathrm{T}$ in EPHA2 and c.356G > A in HSF4. All of the five mutations were screened by targeted NGS for the 38 candidate genes of congenital cataracts, and verified through Sanger DNA sequencing. We confirmed that each mutation cosegregated with the disease phenotypes in the corresponding family and absent in all the unaffected individuals. Further, bioinformatics analysis, conservative prediction and 3-D protein simulation showed that these mutations might be deleterious. According to the ACMG criteria [20], c.1152_1153insG in GJA3 of Family 2 and c.356G > A in HSF4 of Family 5 are clearly pathogenic variants (class V); c.154 $\mathrm{T}>\mathrm{C}$ in GJA8 of Family 1 is a likely pathogenic variant (class IV); c.1804G >C in BFSP1 of Family 3 and c. $1532 \mathrm{C}>\mathrm{T}$ in EPHA2 of Family 4 variants are unknown significance (class III) (Table 3). The unknown significance variants associated with congenital cataracts make them interesting candidates for further studies.

The lens has developed an extensive cell-cell interaction system using connexins to maintain its transparency. Three connexins are expressed in the lens: connexin $43(\mathrm{Cx} 43)$, connexin $46(\mathrm{Cx} 46)$, and connexin 50 (Cx50). Cx43 (GJA1) is expressed mainly in epithelial cells of lens, while Cx46 (GJA3) and Cx50 (GJA8) are expressed in lens fibre cells [21, 22]. GJA8 and GJA3 are the major connexin of the ocular lens, where gap junctions maintain ionic environment, water balance, transparency and optical properties of the lens [23]. To date, 65 variants in GJA8 and 43 variants in GJA3 have been reported in the HGMD (Professional 2019.1) to induce genetic cataracts, which account for about $1 / 4$ of nonsyndromic familial cataract cases. The typical structure of connexin includes cytoplasmic $\mathrm{NH} 2$ - and $\mathrm{COOH}$ - terminal domain, four transmembrane domains and two extracellular loops. The two extracellular loops mediate hemichannel docking between connexons and the E1 loop, which was also shown to be important for the voltage required for closure of gap junction pores [24]. In this study, we identified an amino acid change (F52 L) at the first external loop (E1) in GJA8 in family 1. The altered protein may disrupt normal interactions between the two connexins, which may reduce resistance of the intercellular channel and lead to the leakage of small ions. Moreover, F52 L is highly conserved among many species, so F52 L is very likely to cause disease. In Family 2, frameshift S385Efs*83 in GJA3 resulted from a guanine insertion that introduced a premature translation stop codon located in the $\mathrm{COOH}$-terminus, which may interfere with the folding of the whole protein and resulted in cataract. This insertion mutation (c.1152 1153insG) is similar to the three mutations (c.1137dupC, c.1189dupG, c.1200dupC) reported previously [25-27], thus providing further evidence that the GJA3 Cterminal domain plays an essential role in physiological function of the gene, and further expanding the mutation spectrum of GJA3 in association with congenital cataract.

BFSP1 (filesin) and BFSP2 (phakinin) are major components of the beaded filament, which are unique cytoskeletal lens structures. The biological functions of 


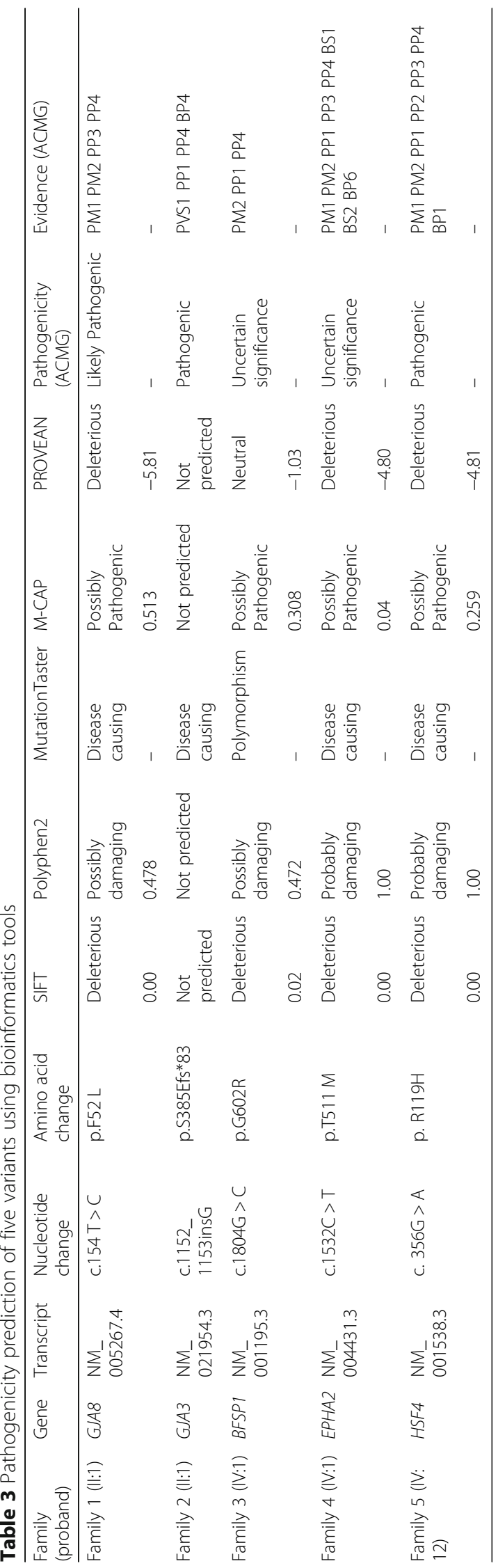




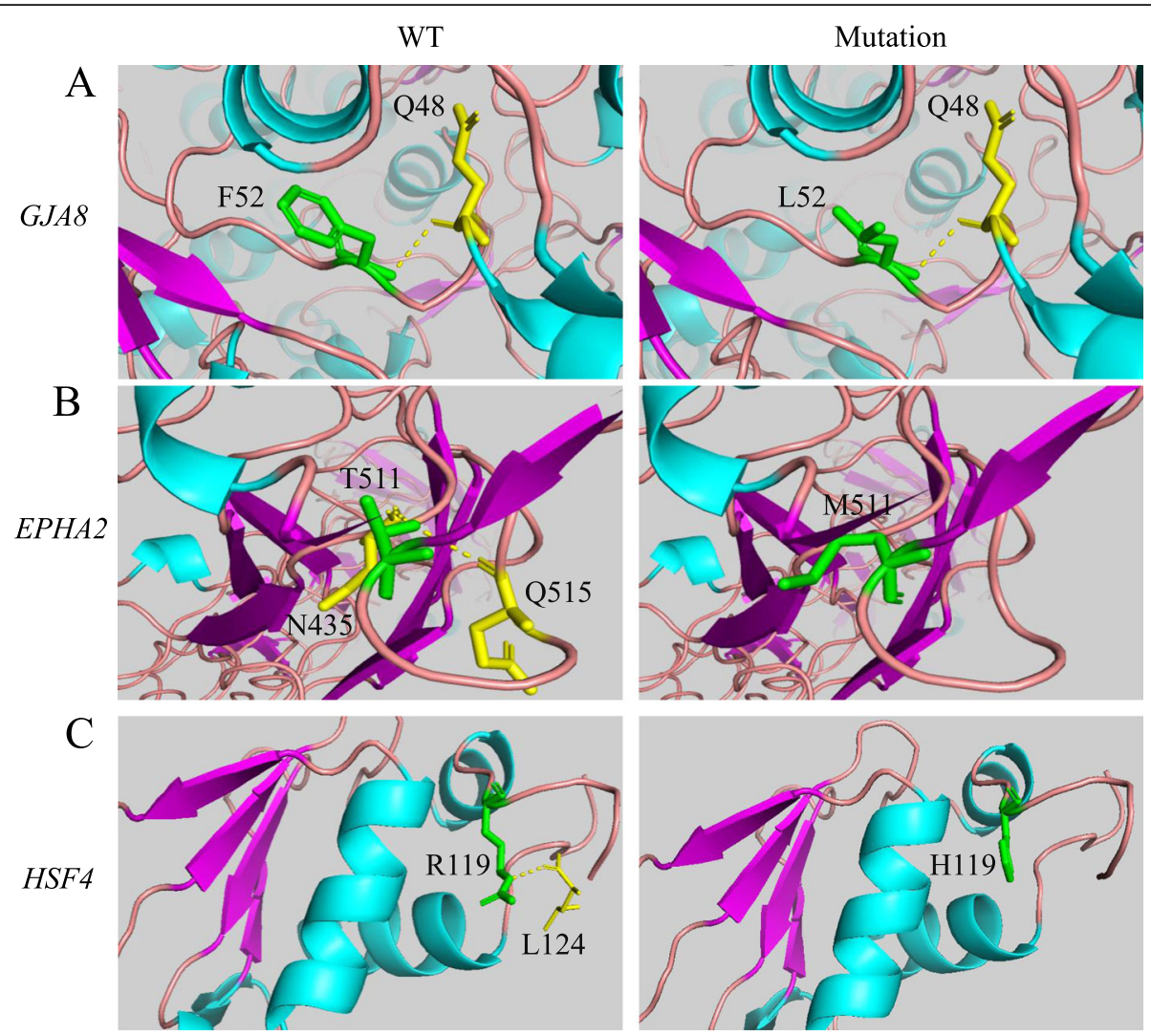

Fig. 5 Three missense mutations (F52 L in GJA8 of Family 1, T511 M in EPHA2 of Family 4 and R119H in HSF4 of Family 5) were simulated by means of SWISS-MODEL and were represented with Ribbon model. The proteins were colored by element: $a$-helix $=b l u e, b$-stand $=$ purple, turn $=$ pink. Wild and mutated amino acids were labeled in green. The amino acids that interacted with the mutation sites with hydrogen bonding were marked in yellow. (a). Substitution of F52 L in GJA8 disturbed the core structure domain and influenced the conformation of the protein. (b). Substitutions of T511 M in EPHA2 destroyed the H-bonding between T511 and N435/Q515. (c). Substitution of R119H in HSF4 destroyed the Hbonding between wild-type R119 and L124

filesin and phakinin are still not clear, but some evidences indicate they play an important role in maintaining lens transparency and homeostasis during fetal development and fiber cell differentiation [28]. A novel mutation c.1804G > C(p.G602R) in BFSP1 was detected in Family 3. Alignment of the BFSP1 protein sequence among different species revealed that the Gly residue at position 602 was less conservative. MutationTaster and PROVEAN prediction tools showed the pathogenicity of G602R was neutral. However, M-CAP, SIFT and PolyPhen 2 analysis indicated that G602R was possibly damaging. Further, mutation was co-segregated with phenotypes in the Family 3 including five affected and four unaffected individuals and that variant frequency was 0.000066 in the ExAC browser, indicating that this variant was rare event in the human genome. Up to now, only six BFSP1 mutations have been reported and four BFSP1 mutations were involved in autosomal recessive cataract families $[11,29,30]$. And two mutations were found in autosomal dominant cataract families. In 2013, Wang et al. first found a heterozygous variant c.1042G $>\mathrm{A}(\mathrm{p} . \mathrm{D} 348 \mathrm{~N})$ in BFSP1 in a 5generation Chinese family in which 15 members had autosomal dominant nuclear cataract [31]. In 2017, Zhai et al. identified heterozygosity for a splice site mutation (c.625 + 3A > G) in BFSP1 in a 4-generation family co-segregating progressive punctate lamellar cataract [32]. The mutation (G602R) highlighted in this study is localized at the tail region of filesin, has an important effect on beaded filament formation as mutation D348N [31]. Taken together with previous research, the results of the Family 3 enriched the suspected pathogenicity of the BFSP1 mutation in human autosomal dominant congenital cataract.

The protein encoded by EPHA2, Ephrin Receptor EphA2, is spatially and temporally regulated in the cortical lens fiber cells, while its expression is lower in anterior epithelial cells, and absent in the nuclei of lens [33]. So far, 22 mutations of EPHA2 have been reported in the patients with congenital cataract, and most of them are in the SAM domain. After identification of p.P584L by Dave et al., we reported the second autosomal dominant 
mutation p.T511 $\mathrm{M}$ in the juxta membrane domain of the protein [34]. The pathogenicity of this mutation was proved in the following three aspects: (1) Protein sequence among different species revealed that the Thr residue at position 511 was highly conserved; (2) Bioinformatics analysis using five prediction tools indicated that $\mathrm{T} 511 \mathrm{M}$ was a pathogenic change. (3) 3-D protein simulation model predicted that amino acids change of M511 T would destroy $\mathrm{H}$-bonding between $\mathrm{T} 511$ interacted via with residues N435 and Q515 of Ephrin type-A receptor 2. Furthermore, this mutation was co-segregated with phenotypes in Family 4. The mutation T511 M identified in EPHA2 gene is a known polymorphism (rs55747232), which raises doubt about its pathogenicity. In conclusion, we believe that M511 T in EPHA2 is a potential variation associated with congenital cataract.

HSF4 belongs to the family of heat-shock transcription factors that bind heat shock elements and activate downstream heat-shock response genes under conditions of stress [35]. It has been reported that HSF4 gene is responsible for both autosomal dominant and autosomal recessive cataracts [36]. We had screened the affected individuals in Family 5 and identified a missense mutation c.356G > A (p.R119H) in HSF4, and this mutation was co-segregated with the disease in all the affected individuals, but not observed in all the unaffected individuals. Protein sequence among different species revealed that the Arginine(R) residue at position 119 is high conserved, and five prediction tools showed p.R119H is pathogenic. 3-D protein simulation predicted that substitution of H119 destroyed the H-bonding, with which wild-type R119 interacted with residues L124 of Heat shock factor protein 4 . Above all provided a persuasive evidence to its pathogenicity of p.R119H in HSF4 of Family 5 .

In summary, we performed genetic analysis in five Chinese families with congenital dominant cataracts and identified five novel mutations, including an insertion mutation encoding p.S385Efs" 83 in GJA3 and four missense mutations: p.F52 L in GJA8, p.G602R in BFSP1, p.T511 $\mathrm{M}$ in EPHA2 and p.R119H in HSF4. This work extended the mutation spectrum of congenital cataracts, and would provide more evidences for the precise diagnosis of the disease.

\section{Conclusions}

We firstly reported five novel mutations associated with autosomal dominant cataracts: c. $154 \mathrm{~T}>\mathrm{C}$ in GJA8, c.1152_1153insG in GJA3, c.1804G $>C$ in BFSP1, c. $1532 \mathrm{C}>\mathrm{T}$ in EPHA2, c.356G $>\mathrm{A}$ in HSF4. This study expands the mutation spectrum of congenital cataracts, and provide solid evidence for genetic counseling and prenatal gene diagnosis of the cataract families.

\section{Supplementary information}

Supplementary information accompanies this paper at https://doi.org/10. 1186/s12881-019-0933-5.

Additional file 1: Table S1. Information of 38 candidate genes for congenital cataract used in targeted NGS.

Additional file 2: Table S2. Variants in five cataract probands from the targeted NGS.

\section{Abbreviations}

BFSP1: Beaded filament structural protein 1; EPHA2: EPH receptor A2; GJA3: Gap junction protein alpha 3; GJA8: Gap junction protein alpha 8; HGMD: Human Gene Mutation Database; HSF4: Heat shock transcription factor 4; M-CAP: Mendelian Clinically Applicable Pathogenicity; NGS: Next generation sequencing; PCR: Polymerase chain reaction; PolyPhen-

2: Polymorphism Phenotyping V2; PROVEAN: Protein variation effect analyzer; SIFT: Scale-Invariant Feature Transform; UCSC: University of California, Santa Cruz

\section{Acknowledgements}

We are very grateful to the family members for their participation in this study, as well as the support of CAMS Innovation Fund for Medical Sciences.

\section{Authors' contributions}

SL conducted experiments, data analysis and drafting the manuscript. JFZ studied the patients and collected the clinical samples. YXC and $Y Y$ analyzed the data. XLZ designed and supervised the study. All authors read and approved the final manuscript.

\section{Funding}

This study was supported by the CAMS Innovation Fund for Medical Sciences (CIFMS) (2016-I2M-3-003) and the National Key Research and Development Program of China (2016YFE0128400 and 2016YFC0905100). The funding bodies played no role in the design of the study and collection, analysis, and interpretation of data and in writing the manuscript.

\section{Availability of data and materials}

The datasets generated and/or analysed during the current study are available in the CNGB Nucleotide Sequence Archive (CNSA: https://db.cngb. org/cnsa; accession number CNP0000764).

\section{Ethics approval and consent to participate}

This study was approved by the Institutional Review Board (IRB) of the Institute of Basic Medical Sciences, Chinese Academy of Medical Sciences, Beijing, China (015-2015). Written consent was obtained from all adult participants and the legal guardians of children under age 18 before they participated in this study.

\section{Consent for publication}

Written consent was obtained from all adult participants and the legal guardians of children under age 18 for the publication of this study, including the images in Fig. 2.

\section{Competing interests}

The authors declare no conflicts of interest.

\section{Author details}

${ }^{1}$ Department of Medical Genetics, Institute of Basic Medical Sciences Chinese Academy of Medical Sciences - School of Basic Medicine Peking Union Medical College, 5 Dong Dan San Tiao, Dongcheng District, Beijing 100005, People's Republic of China. ${ }^{2}$ The No.4 hospital (eye hospital) of Zhangjiakou, Zhangjiakou 075000, People's Republic of China.

Received: 14 May 2019 Accepted: 1 December 2019

Published online: 16 December 2019

\section{References}

1. Hejtmancik JF. Congenital cataracts and their molecular genetics. Semin Cell Dev Biol. 2008;19(2):134-49. 
2. Lambert SR, Drack AV. Infantile cataracts. Surv Ophthalmol. 1996;40(6): $427-58$.

3. Zhou Y, Zhai Y, Huang L, Gong B, Li J, Hao F, Wu Z, Shi Y, Yang Y. A novel CRYBB2 Stopgain mutation causing congenital autosomal dominant cataract in a Chinese family. J Ophthalmol. 2016;2016:1-8.

4. Pichi F, Lembo A, Serafino M, Nucci P. Genetics of congenital cataract. Dev Ophthalmol. 2016;57:1-14.

5. Ionides A, Francis P, Berry V, Mackay D, Bhattacharya S, Shiels A, Moore A. Clinical and genetic heterogeneity in autosomal dominant cataract. Br J Ophthalmol. 1999;83(7):802-8.

6. Vanita, Singh JR, Singh D. genetic and segregation analysis of congenital cataract in the Indian population. Clin Genet. 1999;56(5):389-93.

7. Scott MH, Hejtmancik JF, Wozencraft LA, Reuter LM, Parks MM, Kaiser-Kupfer MI. Autosomal dominant congenital cataract. Interocular phenotypic variability. Ophthalmology. 1994;101(5):866-71.

8. Deng H, Yuan L. Molecular genetics of congenital nuclear cataract. Eur J Med Genet. 2014;57(2-3):113-22.

9. Shiels A, Hejtmancik JF. Genetics of human cataract. Clin Genet. 2013;84(2):120-7.

10. Hilal L, Nandrot E, Belmekki M, Chefchaouni M, El Bacha S, Benazzouz B, Hajaji Y, Gribouval O, Dufier J, Abitbol M, et al. Evidence of clinical and genetic heterogeneity in autosomal dominant congenital cerulean cataracts. Ophthalmic Genet. 2002;23(4):199-208.

11. Ma AS, Grigg JR, Ho G, Prokudin I, Farnsworth E, Holman K, Cheng A, Billson FA, Martin F, Fraser C, et al. Sporadic and familial congenital cataracts: mutational Spectrum and new diagnoses using next-generation sequencing. Hum Mutat. 2016;37(4):371-84.

12. Min HY, Qiao PP, Asan YZH, Jiang HF, Zhu YP, Du HQ LQ, Wang JW, Zhang $J$, et al. targeted genes sequencing identified a novel 15 bp deletion on GJA8 in a Chinese family with autosomal dominant congenital cataracts. Chin Med J. 2016;129(7):860-7.

13. Aoki T, Kunishima S, Yamashita Y, Minamitani K, Ota S. Macrothrombocytopenia with congenital bilateral cataracts: a phenotype of MYH9 disorder with exon 24 Indel mutations. J Pediatr Hematol Oncol. 2018:40(1):76-8

14. Jiang B, Chen Y, Xu B, Hong N, Liu R, Qi M, Shen L. Identification of a novel missense mutation of MIP in a Chinese family with congenital cataracts by target region capture sequencing. Sci Rep. 2017;7:40129.

15. Chen J, Wang Q, Cabrera PE, Zhong Z, Sun W, Jiao X, Chen Y, Govindarajan G, Naeem MA, Khan SN, et al. Molecular genetic analysis of Pakistani families with autosomal recessive congenital cataracts by Homozygosity screening. Invest Ophthalmol Vis Sci. 2017;58(4):2207-17.

16. Chen JH, Qiu J, Chen H, Pang CP, Zhang M. Rapid and cost-effective molecular diagnosis using exome sequencing of one proband with autosomal dominant congenital cataract. Eye. 2014;28(12):1511-6.

17. Liu Y, Wei X, Kong X, Guo X, Sun Y, Man J, Du L, Zhu H, Qu Z, Tian P, et al. Targeted next-generation sequencing for clinical diagnosis of 561 Mendelian diseases. PLoS One. 2015;10(8):e0133636.

18. Miller SADD, Polesky HF. A simple salting out procedure for extracting DNA from human nucleated cells. Nucleic Acids Res. 1988;16(3):1215.

19. Wei X, Ju X, Yi X, Zhu Q, Qu N, Liu T, Chen Y, Jiang H, Yang G, Zhen R, et al. Identification of sequence variants in genetic disease-causing genes using targeted next-generation sequencing. PLoS One. 2011;6(12):e29500.

20. Li Q, Wang K. InterVar: clinical interpretation of genetic variants by the 2015 ACMG-AMP guidelines. Am J Hum Genet. 2017;100(2):267-80.

21. Lamei Yuan* YG, Junhui Yit, Jingjing Xiaoł, Jinzhong Yuant, Wei Xiongt, Hongbo Xu*, Zhijian Yang\$, Jianguo Zhangf, and Hao Dengt: Identification of a Novel GJA3 Mutation in Congenital Nuclear Cataract. Optometry and Vision Science 2015

22. Ge XL, Zhang Y, Wu Y, Lv J, Zhang W, Jin ZB, Qu J, Gu F. Identification of a novel GJA8 (CX50) point mutation causes human dominant congenital cataracts. Sci Rep. 2014:4:4121.

23. Nielsen PA, Baruch A, Shestopalov VI, Giepmans BNG, Dunia I, Benedetti EL, Kumar NM. Lens Connexins alpha 3C $\times 46$ and alpha 8Cx50 interact with zonula occludens protein-1 (ZO-1). Mol Biol Cell. 2003;14(6):2470-81

24. <Exome sequencing identifies novel and recurrent mutations in GJA8 and CRYGD.pdf>.

25. Mackay D, lonides A, Kibar Z, Rouleau G, Berry V, Moore A, Shiels A, Bhattacharya S. Connexin46 mutations in autosomal dominant congenital cataract. Am J Hum Genet. 1999;64(5):1357-64.

26. Zhou D, Ji H, Wei Z, Guo L, Li Y, Wang T, Zhu Y, Dong X, Wang Y, He L, et al. A novel insertional mutation in the connexin 46 (gap junction alpha 3) gene associated with autosomal dominant congenital cataract in a Chinese family. Mol Vis. 2013;19:789-95.

27. Cui XK, Zhu KK, Zhou Z, Wan SM, Dong Y, Wang XC, Li J, Zhang J, Mu HM, Qin $L$, et al. A novel frameshift mutation in CX46 associated with hereditary dominant cataracts in a Chinese family. Int J Ophthalmol. 2017;10(5):684-90.

28. Song S, Landsbury A, Dahm R, Liu Y, Zhang Q, Quinlan RA. Functions of the intermediate filament cytoskeleton in the eye lens. J Clin Invest. 2009;119(7): 1837-48.

29. Ramachandran RD, Perumalsamy V, Hejtmancik JF. Autosomal recessive juvenile onset cataract associated with mutation in BFSP1. Hum Genet. 2007;121(3-4):475-82

30. Li D, Wang S, Ye H, Tang Y, Qiu X, Fan Q, Rong X, Liu X, Chen Y, Yang J, et al. Distribution of gene mutations in sporadic congenital cataract in a Han Chinese population. Mol Vis. 2016;22:589-98.

31. Wang H, Zhang T, Wu D, Zhang J. A novel beaded filament structural protein 1 (BFSP1) gene mutation associated with autosomal dominant congenital cataract in a Chinese family. Mol Vis. 2013;19:2590-5.

32. Zhai Y, Li J, Yu W, Zhu S, Yu Y, Wu M, Sun G, Gong X, Yao K. Targeted exome sequencing of congenital cataracts related genes: broadening the mutation Spectrum and genotype-phenotype correlations in 27 Chinese Han families. Sci Rep. 2017;7(1):1219.

33. Zhang T, Hua R, Xiao W, Burdon KP, Bhattacharya SS, Craig JE, Shang D, Zhao X, Mackey DA, Moore AT, et al. Mutations of the EPHA2 receptor tyrosine kinase gene cause autosomal dominant congenital cataract. Hum Mutat. 2009;30(5):E603-11.

34. Dave A, Laurie K, Staffieri SE, Taranath D, Mackey DA, Mitchell P, Wang JJ, Craig JE, Burdon KP, Sharma S. Mutations in the EPHA2 gene are a major contributor to inherited cataracts in south-eastern Australia. PLoS One. 2013; 8(8):e72518.

35. Bu L, Jin YP, Shi YF, Chu RY, Ban AR, Eiberg H, Andres L, Jiang HS, Zheng GY, Qian MQ, et al. Mutant DNA-binding domain of HSF4 is associated with autosomal dominant lamellar and Marner cataract. Nat Genet. 2002;31(3): 276-8.

36. Forshew T, Johnson CA, Khaliq S, Pasha S, Willis C, Abbasi R, Tee L, Smith U, Trembath RC, Mehdi SQ, et al. Locus heterogeneity in autosomal recessive congenital cataracts: linkage to $9 q$ and germline HSF4 mutations. Hum Genet. 2005;117(5):452-9.

\section{Publisher's Note}

Springer Nature remains neutral with regard to jurisdictional claims in published maps and institutional affiliations.

Ready to submit your research? Choose BMC and benefit from:

- fast, convenient online submission

- thorough peer review by experienced researchers in your field

- rapid publication on acceptance

- support for research data, including large and complex data types

- gold Open Access which fosters wider collaboration and increased citations

- maximum visibility for your research: over $100 \mathrm{M}$ website views per year

At $\mathrm{BMC}$, research is always in progress.

Learn more biomedcentral.com/submissions 